\title{
ДВА АЛГОРИТМА ДЛЯ ВЫЧИСЛЕНИЯ КОНСТАНТ КИСЛОТНОСТИ ДВУХОСНОВНОЙ КИСЛОТЫ
}

\author{
(Представил О. Эйзен)
}

Если константы кислотности двухосновной кислоты отличаются друг от друга на три порядка величины или более, то для их вычисления применяют стандартные процедуры, разработанные для одноосновных кислот $\left[{ }^{1,2}\right]$. Однако часто это условие не выполняется, и рассматриваемые протолитические равновесия перекрываются. Классические методы оценки соответствующих констант 'кислотности $\left(K_{1}\right.$ 'и $\left.K_{2}\right)$, не требующие применения ЭВМ, изложены в монопрафиях $\left[{ }^{1,2}\right]$. С появлением ЭВМ разрабатывались более сложные методы вычисления $K_{1}$ и $K_{2}$ для перекрывающихся протолитических реакций $\left[{ }^{3-5}\right]$. Однако и эти методы рассматривают, как правило, одновременно лишь одну зависимость измеренного параметра от состава среды. Если перекрывающиеся протолитические равновесия иоследуются путем измерения ультрафиолетовых и видимых спектров поглощения, то неизбежно встает вопрос: нельзя ли отказаться от вычисления $K_{1}$ и $K_{2}$ при каждой выбранной длине волны в отдельности и обрабатывать весь спектральный материал одновременно? Этим обеспечивалось бы получение по одному значению для $K_{1}$ и $K_{2}$, которые вместе описывают все изменения в измеренных спектрах. Решению именно этой задачи посвящена настоящая работа.

Рассмотрим два разных алгоритма. Оба они в сущности представляют собой алгоритмы постепенного уточнения первоначально заданных значений $K_{1}, K_{2}, \varepsilon_{A}, \lambda, \varepsilon_{B, \lambda}$ и $\varepsilon_{C}, \lambda$ с целью минимизировать сумму квадратов неувязок в спектрах

$$
Z=\sum_{p=1}^{p=R} \sum_{\lambda=1}^{\lambda=L}\left(\varepsilon_{p, \lambda}-\varepsilon_{p, \lambda}^{\prime}\right)^{2}
$$

где $\varepsilon_{p, \lambda}$ и $\varepsilon_{p, \lambda}^{\prime}$ - измеренный и вычисленный коэффициенты молярного поглощения соответственно для раствора $p$ при длине волны $\lambda$. На каждой длине волны первая протолитическая реакция начинается при $\varepsilon_{\lambda}=\varepsilon_{A, \lambda}$ и кончается при $\varepsilon_{\lambda}=\varepsilon_{B}, \lambda$. Вторая протолитическая реакция начинается при $\varepsilon_{\lambda}=\varepsilon_{B}, \lambda$ и кончается при $\varepsilon_{\lambda}=\varepsilon_{C}, \lambda$. Следовательно, уточнению подлежат $2+3 L=N$ параметров, где $L-$ количество рассматриваемых длин волны. При каждой $\lambda$

$$
\varepsilon_{p, \lambda}^{\prime}=\frac{K_{1} K_{2} \varepsilon_{A, \lambda}+h_{1} K_{2} \varepsilon_{B, \lambda}+h_{1} h_{2} \varepsilon_{C, \lambda}}{K_{1} K_{2}+h_{1} K_{2}+h_{1} h_{2}}
$$

где $h_{1}$ и $h_{2}$ - шкалы кислотности, описывающие первую и вторую протолитические реакции соответственно; в случае сильнокислых сред может быть $h_{1} \neq h_{2}\left[{ }^{6}\right]$. 


\section{Уточнение значений $K_{1}, K_{2}, \varepsilon_{A}, \lambda, \varepsilon_{B}, \lambda$ и $\varepsilon_{C}, \lambda$ \\ на основе алгоритма варьирования коэффициентов уравнения}

Основы алгоритма варьирования коэффициентов уравнения изложены в [7]. Коэффициенты $\left(K_{1}, K_{2}\right.$ и все $\left.\varepsilon_{A}, \lambda, \varepsilon_{B}, \lambda, \varepsilon_{C}, \lambda\right)$ уравнения (2) уточняются поочередным повторением двух циклов. В первом цикле уточняются лишь $K_{1}$ и $K_{2}$ (все $\varepsilon_{A}, \lambda, \varepsilon_{B}, \lambda$ и $\varepsilon_{C}, \lambda$ рассматриваются как постоянные). Во втором цикле, наоборот, уточняются все $\varepsilon_{A}, \lambda, \varepsilon_{B, \lambda}$ и $\varepsilon_{C, \lambda}$ (без изменений $K_{1}$ и $K_{2}$ ). У'казанные циклы повторяются один за другим до тех пор, пока изменения $p K_{1}$ и $p K_{2}$ в двух последующих первых циклах не превысят 0,002 единиц.

\section{Уточнение значений $K_{1}, K_{2}, \varepsilon_{A, \lambda}, \varepsilon_{B, \lambda}$ и $\varepsilon_{C, \lambda}$ методом Гаусса-Ньютона}

Метод Гауоса-Ньютона $[8,9]$ заключается в замене нелинейной функции (2) на каждом шаге линейным приближением

$$
\varepsilon_{p, \lambda}^{\prime \prime}=\varepsilon_{p, \lambda}^{\prime}+\sum_{i=1}^{n=N} \Delta a_{n} \frac{\partial \varepsilon_{p, \lambda}^{\prime}}{\partial a_{n}}
$$

где $\varepsilon_{p, \lambda}^{\prime \prime}$ - вычисленное по уравнению (2) значение $\varepsilon_{p, \lambda}^{\prime}$ с использованием имеющихся значений $K_{1}, K_{2}, \varepsilon_{A}, \lambda, \varepsilon_{B}, \lambda$ и $\varepsilon_{C}, \lambda$, которые в уравнении (3) и далее обозначены через $a_{n} . \Delta a_{n}$ - инкремент коэффициента $a_{n}$ на шаге $j$. На шаге $j+1$

$$
a_{n(j+1)}=a_{n(j)}+\Delta a_{n} .
$$

На каждом шаге итерационной процедуры (4) уточняются значения коэффициентов $a_{n}$ решением системы линейных уравнений

$$
\varepsilon_{p, \lambda}-\varepsilon_{p, \lambda}^{\prime \prime}=0
$$

для получения значений $\Delta a_{n}$. Система уравнений (5) имеет относительно большие размеры: в ней $R \cdot L$ строк и $N=2+3 L$ столбцов. После ее решения методом репрессионного анализа вычисляются по (4) новые, уточненные значения $a_{n}$. Эта процедура повторяется до тех пор, пока значения $p K_{1}$ и $p K_{2}$ на двух последовательных шагах не изменятся больше, чем на 0,001 единиц.

\section{Сравнение работы программ для ЭвМ, составленных на основе рассмотренных выше алгоритмов}

На основе метода варьирования коэффициентов составлена программа УН21, а на базе метода Гаусса-Ньютона - программа УН22. Обе программы составлены на языке Фортран IV и решают одну и ту же задачу - вычисляют $K_{1}$ и $K_{2}$ исходя из ультрафиолетовых и видимых спектров поглощения и кислотности растворов. В том и другом случае необходимо задавать исходные значения для $K_{1}, K_{2}, \varepsilon_{A}, \lambda, \varepsilon_{B, \lambda}$ и $\varepsilon_{C}, \lambda$. Применение вышеуказанных программ для решения тестовых и некоторых реальных задач позволяет сделать следующие выводы:

1. Обе пропраммы дают практически совпадающие результаты.

2. В случае программы. УН21 исходные уточняемые величины могут быть весьма далекими от соответствующих конечных значений. Так, на- 
пример, исходные $K_{1}$ и $K_{2}$ могут отличаться от конечных на $1-2$ порядка величины. Не отмечено ни одного случая, жогда бы процесс итераций, выполняемых по программе УН21, расходился. Однако решение одной и той же задачи по программе УН21 требует больше машинного времени, чем по программе УН22.

3. Программа УН22 нуждается в относительно близких к конечным значениям первоначальных оценках $K_{1}$ и $K_{2}$. Они могут отличаться от конечных лишь в несколько раз. В противном случае процесс итераций может расходиться. Известно, что методу Гаусса-Ньютона свойслвенна медленная сходимость, т. е. требуется больше итераций для стабилизации решения [ $\left.{ }^{9}\right]$. В случае программы УН22 этого не наблюдалось: для достижения требуемой стабильности решения было достаточно выполнить лишь несколько шагов.

Автор выражает благодарность Х. Таал и Р. Юга за оказанную ими помощь при работе с ЭВМ ЕС 1052.

\section{Л ИТ Р РА Т У Р А}

1. Альберт A., Сержент E. Константы ионизации кислот и оснований. М.-Л., 1964, $21-95$.

2. King, E. J. Acid-Base Equilibria. Oxford-London-New York-Paris, 1965, $23-137$.

3. Schwartz, M. L., Gelb, R. I. Statistical analysis of titration data. - Anal. Chem., $1978,50,1571$.

4. Kimberly, M. M., Goldstein, J. H. Determination of $p K_{a}$ values and total proton distribution pattern of spermidine. - Anal. Chem., 1981, 53, 789-793.

5. Motekaitis, R. J., Martell, A. E. Program PKAS: a novel algorithm for the computation of successive protonation constants. - Can. J. Chem., 1982, 60, $168-173$.

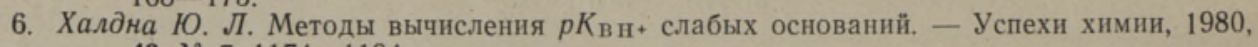
49, № 7, 1174-1194.

7. Халдна Ю. Л. Метод итераций для уточнения коэффнциентов уравнения, предложенного для описания наблюдений. - Уч. зап. Тартуск. ун-та. Труды по химии, 1976, 10, № 2, 118-121.

8. Закускин В. Л. Справочник по численным методам решения уравнений. М., 1960 , $125-130$.

9. Петрович $M$. Л. Регрессионный анализ и его математическое обеспечение на ЕС ЭВМ. М., 1982, 134 .

\section{Ннститут химии \\ Академии наук Эстонской ССР \\ Поступила в редакцию 16/II 1983}

\section{v. HALDNA}

\section{KAKS ALGÓRITMI KAHEALUSELISE HAPPE HAPPELISUSKONSTANTIDE ARVUTAMISEKS}

Esitatud algoritmide alusel koostatud arvutiprogrammid vōimaldavad arvutada kahe omavahel kattuva protolüütilise tasakaalu konstantide väärtused, lähtudes ultravioletsetest neeldumisspektritest ja keskkonna happelisustest. Mōlemal juhul on kasutatud üheaegselt kõiki spektraalandmeid ning minimeeritud eksperimentaalsete ja arvutatud molaarsete ekstinktsioonikoefitsientide vahede ruutude summa, Oks esitatud algoritmidest baseerub vôrrandi koefitsientide varieerimise meetodil ja teine Gauss-Newtoni meetodil mittelineaarsete võrrandisüsteemide lahendamiseks. On võrreldud nimetatud algoritmide alusel koostatud programmide omadusi. 


\section{O. HALDNA}

\section{TWO ALGORITHMS FOR CALCULATING \\ DIPROTIC ACIDS ACIDITY CONSTANTS}

Two algorithms are presented for calculating successive acidity constants of diprotic acids using a set of UV-VIS spectral data and acidities of the solutions. In both cases all spectral data are used simultaneously for calculations; the quantity to be minimized is the sum of squares of deviations between the measured and calculated molar extinction coefficient values. The first algorithm is based on the coefficient variation method, while the second makes use of the Gauss-Newton method of solving systems of nonlinear equations. Characteristic features of the two respective computer programs are compared. 